\title{
Aménagement de l'estuaire de la Gironde
}

\author{
par E. Lespine \\ Ingénieur des Ponts et Chaussées, Directeur de l'Aménagement \\ de l'Estuaire. Port Autonome de Bordeaux
}

\begin{abstract}
Déterminer les effets des aménagements de navigation sur un estuaire serait possible au prix d'une simple observation, si, en l'absence d'aménagement, l'estuaire n'était le siège d'aucune évolution. En fait, il n'en est rien : tous les estuaires sont des milieux extrêmement vivants. L'estuaire de la Gironde, ouvert sur une mer à marée et alimenté par deux fleuves, la Garonne et la Dordogne, n'échappe nullement à la règle. Les fonds y sont, et cela naturellement, en perpétuelle évolution. On le conçoit aisément lorsqu'on sait que les paramètres hydrauliques varient sans cesse : le débit des fleuves et les caractéristiques de la marée. L'évolution des fonds qui en résulte modifie, à son tour, les phénomènes hydrauliques, les courants de marée notamment.

Si donc la détermination a posteriori de l'effet d'un aménagement déterminé sur l'estuaire n'est pas une opération commode, combien plus délicate est encore la prévision des conséquences d'un aménagement projeté. Heureusement, nous disposons aujourd'hui d'outils très puissants, les modèles réduits, pour faire de telles prévisions.
\end{abstract}

\section{Quelques ordres de grandeur}

Avant d'aborder quelques exemples, propres à la Gironde, de réactions de l'estuaire à certaines interventions humaines ou de prévisions de réaction pour des aménagements projetés, je crois qu'il faut citer quelques ordres de grandeur des phénomènes naturels en cause :

1) A chaque marée de vive eau, deux milliards de mètres cubes d'eau rentrent dans l'estuaire au niveau de Pointe de Grave-Royan. Le volume d'eau oscillant n'est plus que de 250 millions de mètres cubes environ au niveau du bec d'Ambès et il est réduit à 80 millions de mètres cubes à $100 \mathrm{~km}$ de la Pointe de Grave, dont les deux tiers environ pour la Garonne, un tiers pour la Dordogne.

2) Le débit de la Garonne varie entre $70 \mathrm{~m}^{3} / \mathrm{s}$ en étiage et 6000 à $8000 \mathrm{~m}^{3} / \mathrm{s}$ en crue et celui de la Dordogne reste inférieur à $2000 \mathrm{~m}^{3} / \mathrm{s}$. Quant au débit solide annuel des deux fleuves, il est de l'ordre de 2 millions de tonnes, dont les trois quarts pour la Garonne et le quart pour la Dordogne.

3) Le poids de sédiment traversant, à l'état de suspension, une section aval de l'estuaire en une marée est de l'ordre de 1 million de tonnes.

4) Le chenal de grande navigation est une bande étroite de 200 ou $300 \mathrm{~m}$ de largeur dans un estuaire dont la largeur varie entre $2000 \mathrm{~m}$ et $10000 \mathrm{~m}$ environ.

5) Le gonflement de l'onde marée résultant, au cours de sa remontée, du rétrécissement progressif de l'estuaire est atténué à cause des frottements sur les rives d'autant plus importants que le coefficient de marée est plus élevé : Ainsi le marnage qui passe, par marée de coefficient 40 , de $2,30 \mathrm{~m}$ au Verdon à $4 \mathrm{~m}$ à Bordeaux passe seulement de $5 \mathrm{~m}$ au Verdon par marée de coefficient 110 à $5,50 \mathrm{~m}$ environ à Bordeaux.

6) Entre 1939 et 1949 , période où les interventions humaines ont été très réduites, les évolutions naturelles des fonds ont porté sur plusieurs dizaines de millions de mètres cubes.

\section{Exemples d'aménagements réalisés et leurs effets}

Choisissons à présent quelques aménagements réalisés sur la Gironde depuis un siècle environ et analysons leurs effets sur les fonds. 
Comme indiqué en préambule, il n'est pas facile de faire la distinction entre les évolutions de l'estuaire résultant de laménagement considéré et celles consécutives à la variation naturelle des paramètres hydrauliques. De plus, des variations saisonnières des fonds peuvent masquer partiellement une évolution continue. Ce ne sont donc que les mouvements importants des fonds décelés sur une longue période qui sont pris en considération.

\section{L'aménagement de la Garonne en amont du bec d'Ambès}

Vers 1850 , la Gironde offrait à la navigation des profondeurs sours étiage de l'ordre de $7 \mathrm{~m}$ jusqu'au droit de Goulée, de 3 à $4 \mathrm{~m}$ jusqu'au bec d'Ambès et de $1 \mathrm{~m}$ à $1,20 \mathrm{~m}$ environ en amont du bec d'Ambès. Malgré la surcote de la marée, qui conduisait alors à un tirant d'eau autorisé à Bordeaux de l'ordre de $5 \mathrm{~m}$, les premiers travaux d'amélioration des accès ont été jugés nécessaires.

Le premier souci a été d'éviter les divagations du chenal : C'est ainsi qu'a été raccordée à la terre l'île de Grattequina (cf. fig. 1). La construction d'une digue insubmersible à Bassens a permis de faire coïncider l'action du flot et l'action du jusant. Un approfondissement durable de $2 \mathrm{~m}$ a été obtenu.

La même idée a conduit à réaliser, dès 1860 , à l'extrémité amont du bras de Macau, une digue longitudinale en deux tronçons séparés par une brèche de $800 \mathrm{~m}$, le tronçon amont étant arasé à $+2,50 \mathrm{~m}$ et le tronçon aval à $+1,20 \mathrm{~m}$ et un barrage transversal arasé à la cote - $3 \mathrm{~m}$ environ (cf. fig. 2). Mais ces ouvrages, complétés par un éperon en prolongement du bec d'Ambès, ne se sont pas avérés suffisants pour assurer la coïncidence des actions de flot et de jusant. Des épis ont dû être aménagés des deux côtés. De plus, les ouvrages de Macau ont été surélevés à plusieurs reprises et, en dernier lieu en 1961, à des cotes soigneusement étudiées sur modèle au Laboratoire National d'Hydraulique de Chatou. Il s'agissait, en jouant sur la dissymétrie existant dans la courbe de marée, de gêner le moins possible le passage du flot par le bras de Macau tout en s'opposant au maximum à l'écoulement du jusant par ce même bras. Les résultats ont été spectaculaires : les fonds se sont maintenus à la cote $-3,00 \mathrm{~m}$ dès la fin de la construction des épis vers 1900. Dragués depuis à une cote comprise entre -6 et $-7,00 \mathrm{~m}$, ils se maintiennent à présent de façon particulièrement remarquable.

Il est intéressant de noter par contre l'inefficacité totale d'un dragage, massif pour l'époque, entrepris à Bassens vers 1890 qui avait consisté à ouvrir un chenal de $50 \mathrm{~m}$ de largeur à la cote $-4 \mathrm{~m}$ environ, lequel, durant les basses eaux, se comblait avec de la vase. Deux ans après le travail, un exhaussement de $1,50 \mathrm{~m}$ environ avait ramené pratiquement la cote à sa valeur initiale, soit $-2,50 \mathrm{~m}$.

\section{La digue de Valeyrac}

Un autre exemple dans une zone où, comme nous l'avons dit, les phénomènes naturels sont d'une toute autre ampleur, est celui de la digue de Valeyrac. Alors que, jusqu'en 1920 , les cotes naturelles -3 à $-4 \mathrm{~m}$ existant en Gironde avaient permis de très larges développements du trafic maritime du port de Bordeaux, la nécessité de mettre à la disposition de la navigation maritime un chenal stable et adapté aux caractéristiques des navires de l'époque justifiait une intervention dans la partie aval de l'estuaire. Une digue en graviers de $2 \mathrm{~m}$ de hauteur environ et de $10 \mathrm{~km}$ de longueur a été construite, vers 1925 , dans le but de guider les courants, sans gêner la pénétration du flot (cf. fig. 3). Dix ans plus tard, M. François Lévêque, constatant que cet ouvrage n'avait pas eu d'influence sensible sur les fonds, décidait d'ouvrir un chenal à la cote $-6,50 \mathrm{~m}$ à près de $2 \mathrm{~km}$ de distance de la digue, c'est-à-dire d'assurer artificiellement, par dragage, la continuité des fosses de flot et de jusant. Les nouvelles profondeurs ont pu être maintenues pendant plusieurs années au prix de dragages limités. Actuellement, toutefois, ce secteur, où la profondeur a été portée à $-7 \mathrm{~m}$ environ, fait l'objet d'études minutieuses, car c'est là que doit être à présent exécutée une partie importante des dragages d'entretien du chenal intérieur. L'augmentation du volume de dragages dans ce secteur semble tenir davantage à une diminution de la puissance érosive du jusant, consécutive à l'ouverture d'une brèche en amont dans le banc de Saint-Estèphe, qu'à un creusement important des fonds qui, avant intervention, se trouvaient à la cote - $4 \mathrm{~m}$ environ.

II est bon de noter que, malgré cet échec de la digue de Valeyrac, l'ensemble des ouvrages réalisés en Gironde a permis de gagner 3,4 , voire $5 \mathrm{~m}$ en certains points sur la profondeur du chenal d'accès à Bassens et Bordeaux, mais que, dans le même temps, les sections moyennes de l'estuaire n'ont pas varié de façon significative.

\section{La passe d'entrée en Gironde}

Parni les réactions observées de l'estuaire à des interventions humaines je prendrai un dernier exemple, celui de la passe d'entrée en Gironde : les profondeurs dans la passe naturelle existante étant jugées insuffisantes en 1930 , une passe fut créée artificiellement, par dragages, par la première drague aspiratrice en marche, la drague $P$. Lefort, engin capable d'intervenir par houle d'un creux pouvant atteindre $2 \mathrm{~m}$. Dix millions de mètres cubes ont ainsi été prélevés, on peut dire en pleine mer. La nouvelle passe à la cote $-9,50 \mathrm{~m}$ (cf. fig. 4) permettait alors la réception au Verdon des plus grands navires à flot ou en construction. Sa stabilité s'est avérée particulièrement remarquable, puisqu'elle est encore en service actuellement, et même à la cote $-13,50 \mathrm{~m}$. L'on peut dire que ce dragage pourtant important n'était pas à l'échelle des phénomènes naturels et qu'il ne pouvait donc pas avoir d'influence sur eux : 200 millions de mètres cubes de sable se sont accumulés sur le banc de la Coubre depuis 1930. Mais inversement, tracée de façon particulièrement judicieuse, la passe n'a pas été menacée par ces évolutions naturelles fort importantes.

\section{Améliorations prévues}

Le Port de Bordeaux, voulant jouer à présent à fond les deux cartes maîtresses que lni offre la nature, celle d'un port d'estuaire à $100 \mathrm{~km}$ de la mer accessible par une très grande voie d'eau et celle d'un grand port implanté 

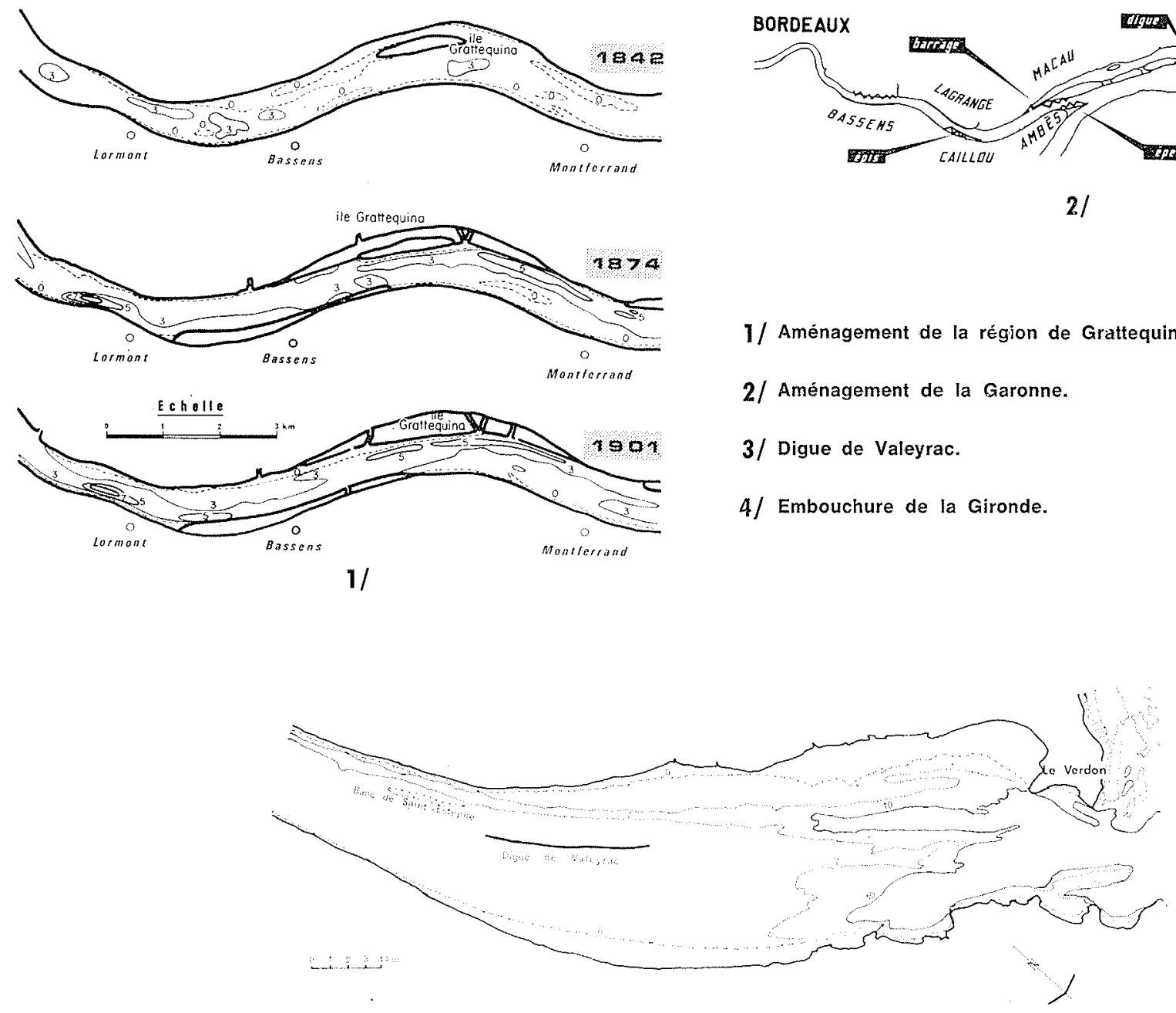

3/

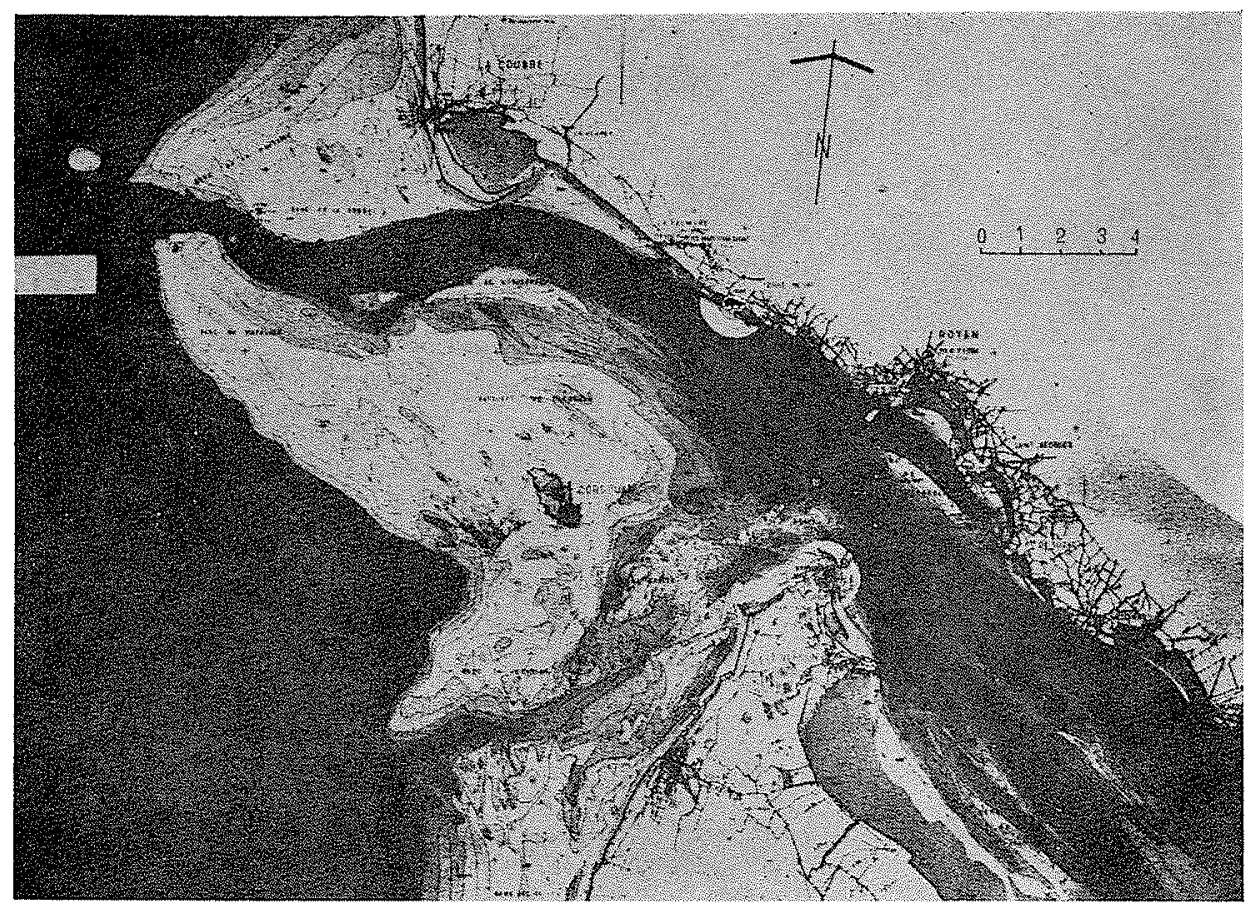

4/



21

1/ Aménagement de la région de Grattequina.

2/ Aménagement de la Garonne.

3/ Digue de Valeyrac.

4/ Embouchure de la Gironde. 


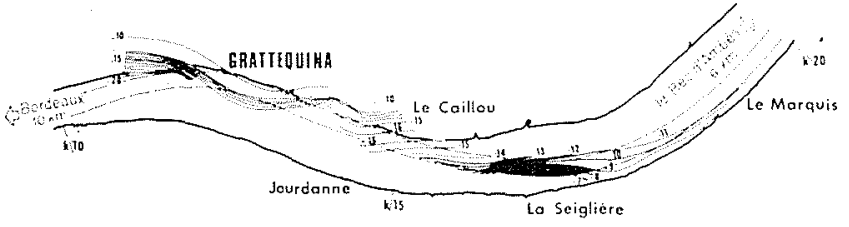

REGION DU CAILLOU
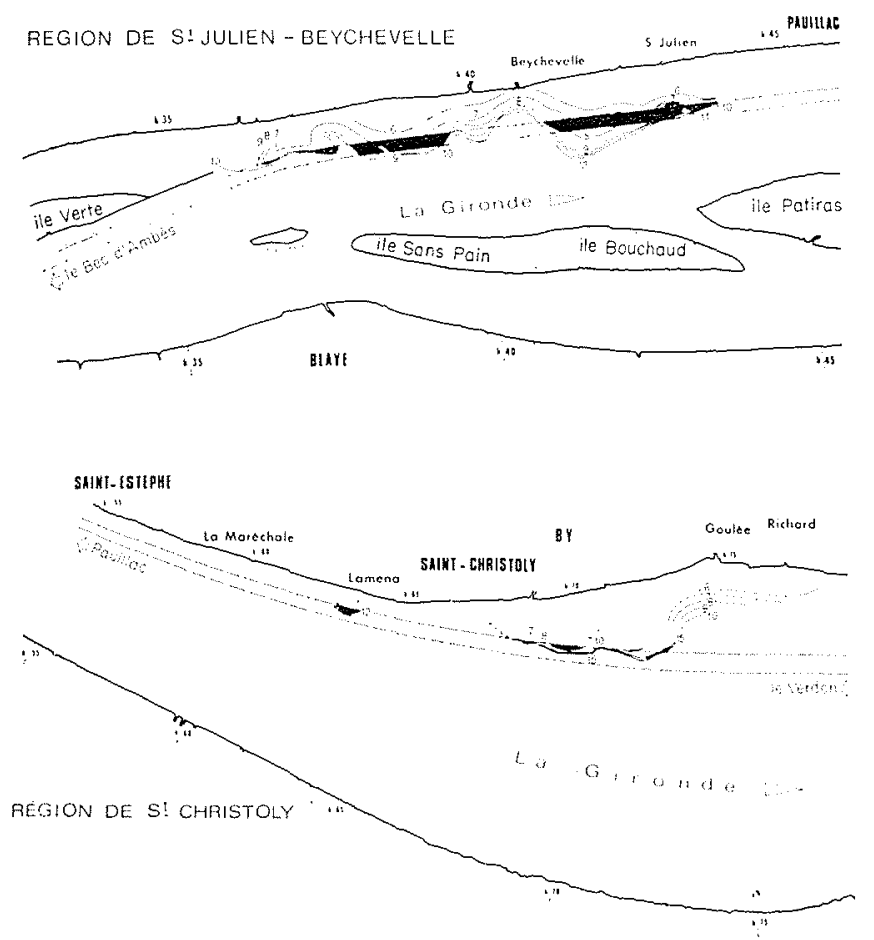

5/ Estuaire de la Gironde. Seuils rocheux. sur le littoral, accessible aux plus grands bateaux en service ou en construction, de nouvelles améliorations importantes des profondeurs du chenal d'accès sont envisagées à court terme :

- $6 \mathrm{~m}$ pour le chenal d'accès au Verdon;

- $2 \mathrm{~m}$ pour le chenal d'accès intérieur, fréquenté d'ores et déjà par des navires de $10 \mathrm{~m}$ de tirant d'eau par marée moyenne et bientôt par toutes marées.

Ceci a conduit tout naturellement le Port Autonome de Bordeaux à se demander quelles seraient les réactions de l'estuaire à de tels aménagements. Pour prévoir ces réactions, il a mis en œuvre, en collaboration avec divers laboratoires et principalement le Laboratoire National d'Hydraulique de Chatou, un programme d'études très important que je présente sommairement.

\section{Les mesures en nature préliminaires}

De très nombreuses observations en nature ont été effectuées dans le but de connaître les mécanismes sédimentaires essentiels et de recueillir les données indispensables à l'étalonnage de modèles réduits.

En premier lieu, des levés fréquents des fonds sont venus compléter la collection impressionnante et fort précieuse de plans de sondages, collection disponible en raison de l'importance qui a été accordée, depuis plusieurs siècles, à cette voie exceptionnelle de pénétration à l'intérieur des terres que constitue l'estuaire girondin.

L'on a, par ailleurs, relevé plusieurs fois au cours d'une marée complète et en cinq points de l'estuaire, aux P.K. $54,67,71,78$ et 89 , les distributions, sur toute la verticale, des courants, de la salinité et de la turbidité. Une interprétation très brillante de toutes ces mesures a été effectuée par le Laboratoire de Chatou.

Plusieurs expériences de traceurs radioactifs nous ont

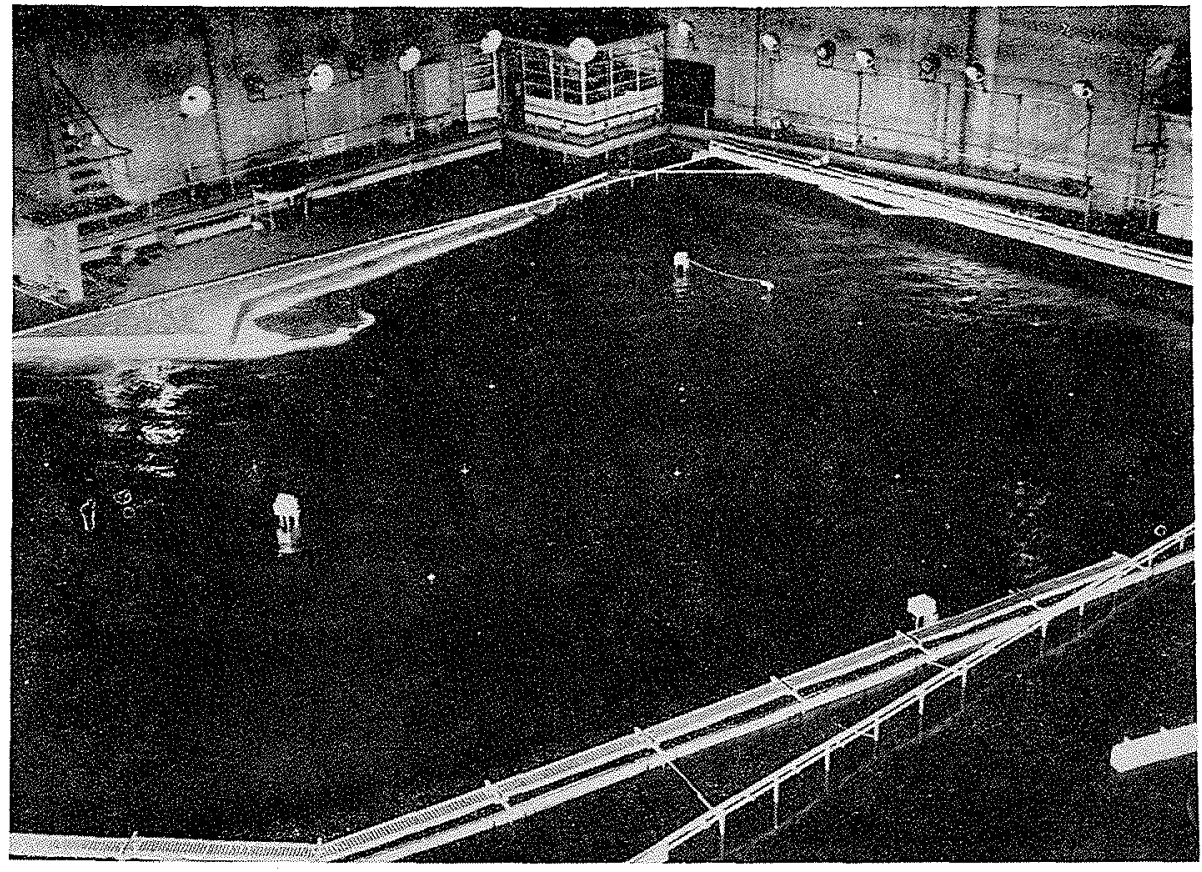

6/ Modèle de l'embouchure. 
enfin apporté des indications fort intéressantes sur les mouvements des sables et même des vases.

\section{Les modèles mathématiques}

Des modèles mathématiques, étalonnés sur quelques données naturelles, donnent une vue synthétique de l'hydraulique de l'estuaire et de l'embouchure et montrent l'influence du débit fluvial sur les conditions de propagation de la marée. Nous espérons que sera bientôt mis au point un modèle mathématique de turbidité intégrant les lois d'érosion, de transport en suspension et de dépôt des vases, mises en évidence sur une installation originale réalisée au Laboratoire National d'Hydraulique : «le tube à vase » (Banc d'essai de la dynamique des sédiments fins cohésifs).

\section{Les modèles réduits}

N'étant pas parvenus à une compréhension suffisante de la dynamique sédimentaire, nous avons eu recours très largement à la technique des modèles réduits pour définir les conditions d'approfondissement du chenal (tracé en plan) et les ouvrages de calibrage éventuellement nécessaires pour que la réaction de l'estuaire soit favorable, c'est-à-dire qu'il n'y ait pas tendance à comblement rapide du chenal approfondi.

En fait, pour ce qui concerne le chenal intérieur entre Le Verdon et Bordeaux, son tracé a été pratiquement fixé, avant l'étude sur modèle, lorsqu'au terme d'une campagne de reconnaissance très importante par vibrofonçage on a constaté que l'approfondissement du chenal actuel ne nécessiterait l'arasement que de trois seuils rocheux de faible emprise (cf. fig. 5) et que le respect du tracé actuel s'avérerait en conséquence beaucoup plus économique que toute autre solution visant à contourner les seuils rocheux et à mieux utiliser le chenal antéflandrien.
Pour l'embouchure, au contraire, le tracé en plan ne pouvait pas être fixé a priori. Compte tenu en effet de l'approfondissement très important envisagé $-6 \mathrm{~m}$ - et donc du coût relativement peu élevé d'abandon du tracé actuel, seul un calcul économique intégrant le coût de l'approfondissement et le coût d'entretien pendant plusieurs années - dont l'ordre de grandeur peut être avancé grâce précisément aux études sur modèle — pouvait conduire à la sélection de la meilleure solution.

\section{Etude de l'approfondissement de la passe d'entrée à la cote $-19,50 \mathrm{~m}$}

Cette étude a été effectuée sur un modèle de $860 \mathrm{~m}^{2}$ environ représentant l'embouchure de la Gironde depuis la ligne Pointe de Grave-Royan jusqu'à l'isobathe $(-25 \mathrm{~m})$ au large, à l'échelle du $1 / 1250^{\circ}$ en plan et du $1 / 100^{\circ}$ en hauteur (cf. fig. 6).

L'étalonnage hydraulique a consisté à rendre les champs de courants aussi conformes que possible aux données naturelles.

Le matériau mobile, styvarène de masse volumique $1,04 \mathrm{~g} / \mathrm{cm}$ et de $D_{50}$ égal à $1,5 \mathrm{~mm}$, a été choisi en raison de conditions d'entraînement par les courants de marée représentant de façon correcte celles du sable en nature. Toutefois, du fait de la distorsion, cet entrainement par les courants n'était satisfaisant que pour une hauteur d'écoulement comprise entre 5 et $15 \mathrm{~m}$ environ. De façon plus précise, le sommet des bancs était trop mobile et les matériaux dans le fond des chenaux trop stables.

Les caractéristiques de la houle ont été ajustées sur le modèle de telle façon que la mobilité par la houle soit correctement représentée. Le creux de la houle a dû être réduit huit fois par rapport aux hauteurs, ce qui excluait bien entendu la représentation du transport littoral, phénomène en réalité secondaire dans le processus d'évolution des fonds de l'embouchure.



7/ Diverses solutions éludiées. 


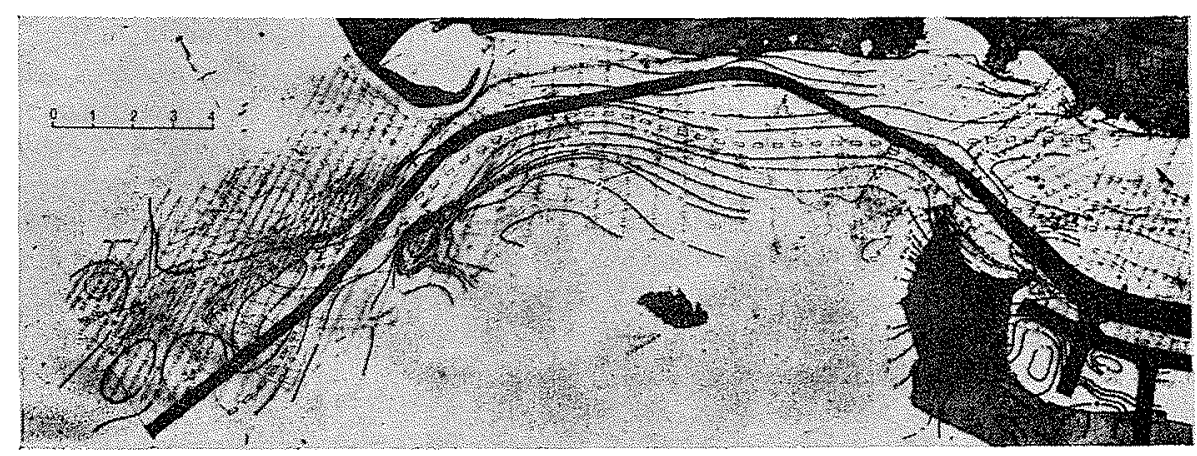

8/ Accès à la zone industrialo-portuaire du Verdon.

L'étalonnage sédimentologique a porté sur les périodes 1874-1930 et 1932-1965. Dix marées sur le modèle, durant chacune 6 minutes, soit 1 heure sur le modèle, représentent une année d'évolution en nature.

Parmi toutes les solutions étudiées, six se sont avérées particulièrement intéressantes (cf. fig. 7). Le choix de la solution définitive (cf. fig. 8) a été effectué au terme d'une étude très fine portant :

— sur la stabilité de la passe;

— sur les facilités de navigation;

- sur le coût global (approfondissement + entretien) actualisé des diverses passes.

La valeur de la solution retenue provient essentiellement du fait qu'elle se situe à la limite de la zone soumise à la plus forte évolution. Les études géologiques du site et sédimentologiques sur modèle ont montré qu'il serait parfaitement possible de réaliser plusieurs approfondissements successifs ultérieurs sur le même tracé, en vue de recevoir un jour, si nécessaire, en rade du Verdon des navires de $30 \mathrm{~m}$ de tirant d'eau (cf. fig. 9).

\section{Etude de l'aménagement de l'estuaire pour la réception de navires de 11 à $12 \mathrm{~m}$ de tirant d'eau}

Cette étude a été menée - elle n'est pas achevée — sur plusieurs modèles :
- UN MODÈLE D'ENSEMBLE à fonds fixes représente, à l'échelle du $1 / 2000^{\circ}$, en plan la Gironde, la Garonne jusqu'à La Réole et la Dordogne jusqu'à Pessac (cf. fig. 10). La marée dure $5 \mathrm{mn} 15 \mathrm{~s}$ sur le modèle.

Le tracé du chenal étant imposé, l'étude a consisté simplement à rechercher les ouvrages susceptibles d'améliorer l'auto-curage du chenal par les courants. Il fallait rechercher un critère purement hydraulique traduisant la tendance du chenal à être plus ou moins stable. Après diverses recherches, il a été décidé de retenir le paramètre :

$$
\frac{\mathrm{J}-\mathrm{F}}{\mathrm{T}}
$$

$\mathrm{J}$ étant le volume d'eau traversant $1 \mathrm{~m}^{2}$ de section au droit du chenal pendant le jusant;

$F$ étant le volume d'eau traversant $1 \mathrm{~m}^{2}$ de section au droit du chenal pendant le flot.

L'on a toutes raisons de penser que l'aménagement est d'autant meilleur que la valeur de ce paramàtre est plus élevée.

Les champs de courants relevés à divers moments de la marée ont été également pris en considération pour la sélection des meilleurs variantes d'aménagement.

DeuX MOdÈles PARTIELS A FONDS MOBILES :

- celui de "Saint-Christoly», représentant la partie aval de l'estuaire, de Lamena (PK. 63) à Pointe de Grave

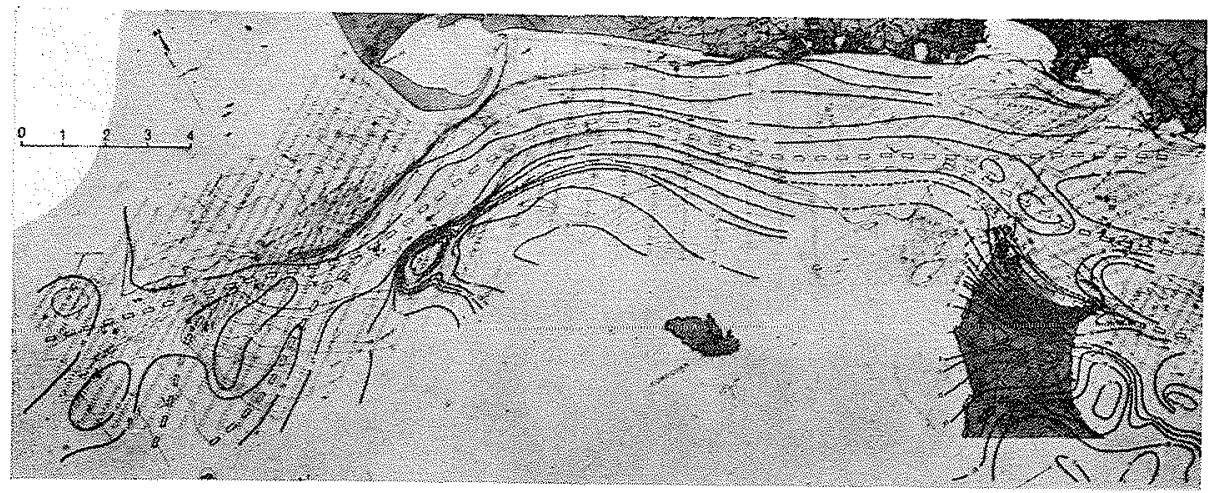

9/ Cartes des isobathes du toit du fond rocheux 
(PK. 96), à l'échelle du $1 / 600^{\circ}$ en plan et $1 / 60^{\circ}$ en hauteur (cf. fig. 11).

- et celui «des Iles», représentant l'estuaire depuis l'île Verte (PK. 30) jusqu'à La Maréchale (PK. 60), à l'échelle du $1 / 400^{\circ}$ en plan et $1 / 60^{\circ}$ en hauteur : sa longueur est de l'ordre de $75 \mathrm{~m}$ (cf. fig. 12).

Les études ont consisté :

- Dans une première phase - les modèles étant encore à fonds fixes - à regarder, en quelque sorte à la loupe, les effets produits par les aménagements sélectionnés sur le modèle d'ensemble, les conditions aux limites à réaliser sur les modèles partiels ayant préalablement été relevées sur le modèle d'ensemble.

- Dans une deuxième phase, les fonds des modèles étant rendus mobiles, à ajuster les caractéristiques des ouvrages en fonction des mouvements des fonds observés.

Par les études approfondies menées sur ces trois modèles, on a pu mettre en évidence trois types d'ouvrages de calibrage (cf. fig. 13) :

- des ouvrages de liaison entre les îles complétés par une digue prolongeant l'îlot de Trompeloup et rétablissant ainsi la continuité du banc de Saint-Estèphe, améliorent l'autocurage du chenal depuis les îles (PK. 35) jusqu'au PK. 65;

- un barrage submersible arasé à la cote $+1 \mathrm{~m}$ en aval de Blaye améliore sensiblement la situation du chenal jusqu'au PK. 60;

- des épis en rive droite, implantés aux PK. 65, 71, 76, améliorent sensiblement, lorsqu'ils sont seuls - c'est-àdire, sans le barrage de Blaye - la situation à l'aval de l'estuaire. Là apparaît clairement le principe de solidarité selon lequel il est impossible d'étudier de façon isolée un tronçon de l'estuaire. Il est, par ailleurs, tout à fait remarquable que ces épis, de longueur modeste, $1 \mathrm{~km}$ à $1,5 \mathrm{~km}$, aient un effet aussi bénéfique, eu égard à l'importance des phénomènes naturels en jeu.

\section{Etude de l'aménagement}

\section{de la zone industrialo-portuaire du Verdon}

Le Port de Bordeaux ayant, parmi les ports de l'Atlantique, la possibilité de jouer la carte du gigantisme naval, il pouvait prétendre, du même coup, avoir une vocation à accueillir des industries lourdes, grandes consommatrices de matières premières et grandes exportatrices de produits finis. Ainsi est née, il y a quelques années, l'idée de créer en amont du Verdon une vaste zone industrialo-portuaire de 10000 ha, en partie gagnée sur l'estuaire (cf. fig. 8). Les études géologiques ayant montré la possibilité d'aménager un tel port avec darses à grandes profondeurs, l'avant-projet a été aussitôt étudié sur modèle réduit :

- d'une part, dans le but de déterminer les réactions de l'estuaire à cet aménagement fort important;

- d'autre part, en vue de définir la meilleure configuration des ouvrages pour rendre l'exploitation des nouvelles installations aussi confortables que possible.

Dans ce deuxième but, nous avons construit un modèle à l'échelle du $1 / 200$ " sur lequel évoluera une maquette de pétrolier de $250000 \mathrm{tpl}$.

Pour la prévision des réactions de l'estuaire, l'on a utilisé tout naturellement les modèles mis en place pour l'étude

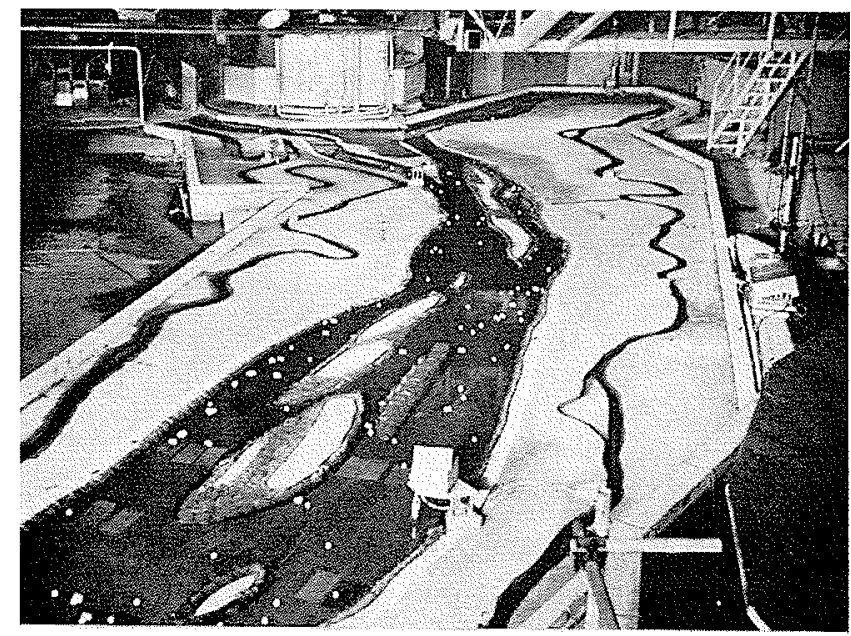

10) Modèle d'ensemble.

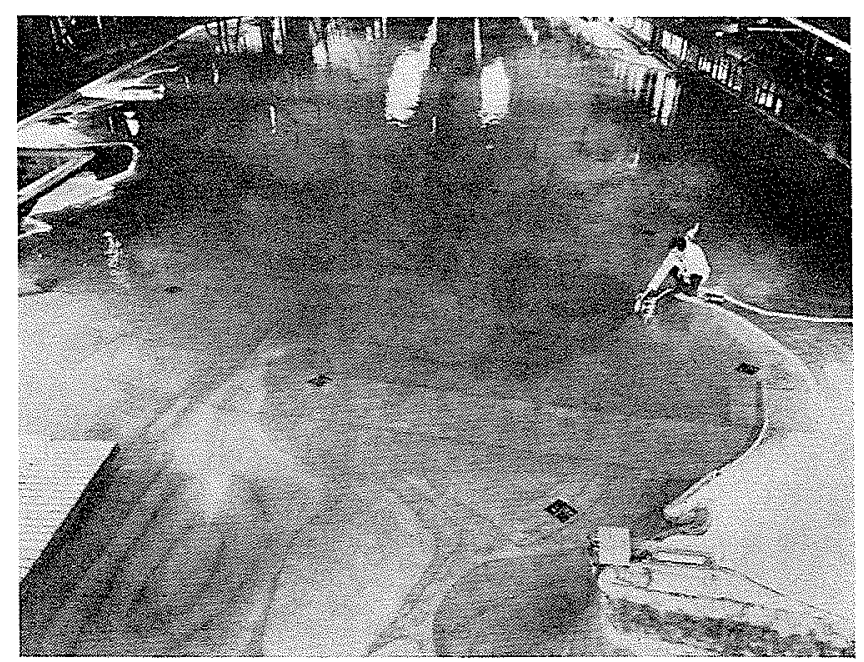

11/ Modèle de Saint-Christoly.

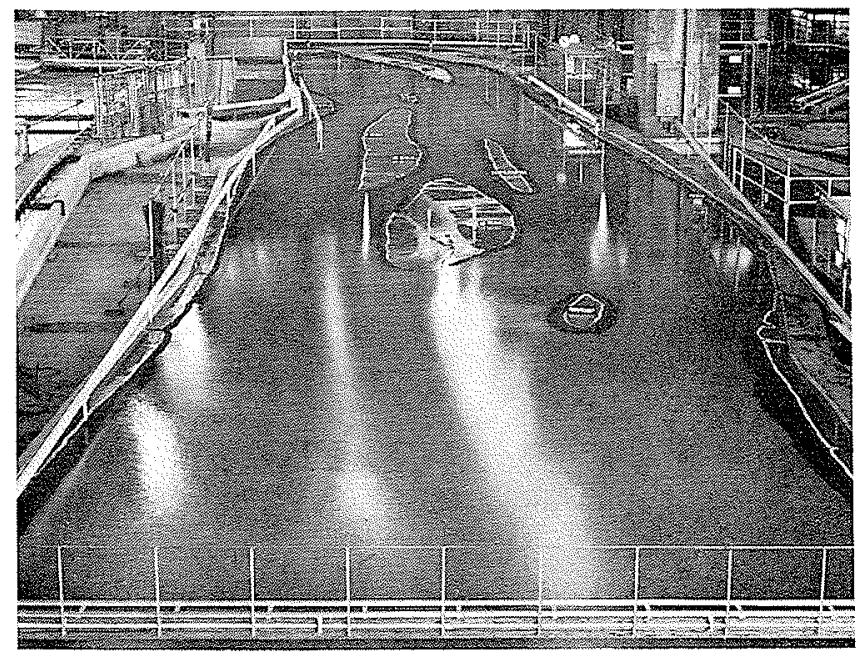

12/ Modèle des îles. 


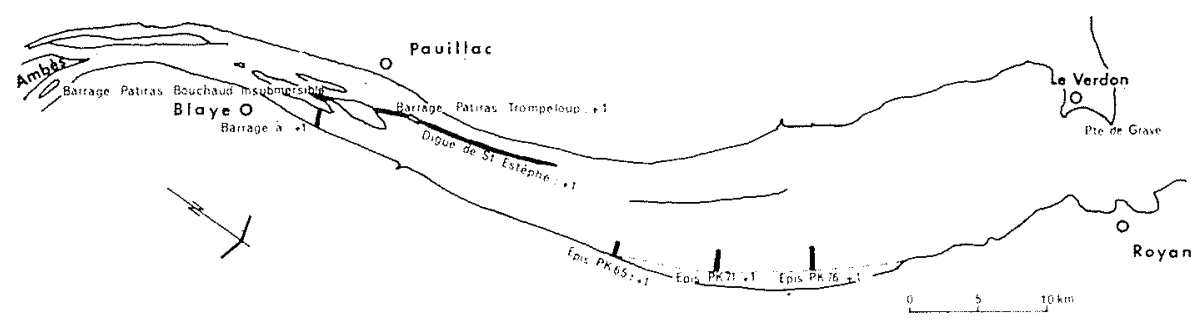

13/ Plan d'aménagement de l'estuaire.

du chenal de grande navigation. Le problème a été dégrossi sur le modèle d'ensemble. L'étude est en cours d'achèvement sur le modèle de Saint-Christoly.

Sur ce même modèle, ont également été définis les ouvrages qui constitueront, dès la fin du $\mathrm{VI}^{\mathrm{e}} \mathrm{Plan}$, une première étape de ce vaste ensemble industrialo-portuaire. C'est ainsi qu'ont été définies la meilleure implantation et les caractéristiques :

- d'une digue d'appui des courants de $910 \mathrm{~m}$ de longueur;

- d'une première tranche de remblai de 300 ha;

- et d'un deuxième poste d'accostage pour grands pétroliers.

L'on a effectué des mesures nombreuses d'intensité et de cap de courants et d'efforts sur une plaque figurant un pétrolier dans la phase préparatoire à l'accostage.

\section{Conclusions}

En conclusion, l'on peut dire :

- que l'analyse a posteriori des effets d'un aménagement donné est fort compliquée du fait de la variation perpétuelle de tous les facteurs et des interventions humaines (dragages notamment);
- que les aménagements de la partie fluvio-maritime qui se sont avérés les plus efficaces sont ceux qui respectaient quelques principes élémentaires, tels que coïncidence des actions de flot et de jusant, variation aussi régulière que possible des largeurs et des sections mouillées moyennes, utilisation optimale de la puissance érosive du jusant en barrant des bras secondaires par des ouvrages submersibles ne s'opposant pratiquement pas à la pénétration du flot, mais favorisant l'écoulement du jusant dans le bras principal..

- que, malgré l'importance des phénomènes naturels en cause, des ouvrages relativement modestes peuvent modifier sensiblement la répartition transversale de l'écoulement, dans la partie fluvio-maritime tout au moins;

- que si, dans la partie fluvio-maritime, des ouvrages de calibrage sont nécessaires pour réduire le volume des dragages d'entretien consécutifs à l'approfondissement du chenal, dans l'embouchure, par contre, le volume de dragages d'entretien est en première approximation indépendant de la cote du chenal, mais bien plus fonction du tracé de la passe;

- que la technique des modèles réduits nous offre, aujourd'hui, le moyen de prévoir, avec le maximum de chances de succès, les réactions de l'estuaire aux aménagements souvent fort importants répondant aux besoins de la navigation, du commerce et des industries portuaires.

\section{Discussion}

Président : M. J. CHAPON

M. le Président remercie chaleureusement M. Lespine de son brillant exposé et ouvre la discussion.

M. Maquet (Port Autonome de Nantes-Saint-Nazaire) demande si les conditions de sédimentation ont été étudiées dans les darses projetées en bordure du chenal du Verdon.

Pour éviter des dépôts massifs dans les darses, on a placé l'unique entrée vers celles-ci, en un point de l'estuaire où la turbidité des eaux présente une brusque diminution, répond M. LEspine.

En l'absence d'autre demande d'intervention, $M$. le Président clôt la discussion et donne la parole à M. MAQueT pour l'exposé de sa communication. 